\title{
Making a Good (Virtual) First Impression: The Use of Visuals in Online Impression Management and Creating Identity Performances
}

\author{
Erika Pearson \\ Department of Media, Film and Communication, University of Otago, \\ P.O. Box 56, Dunedin, New Zealand \\ erika.pearson@otago.ac.nz
}

\begin{abstract}
This paper argues that visual elements are becoming an increasingly important component of identity performance on social networking sites. Working from a narrative approach to identity, this paper explores how images on SNS are used both as part of an impression management strategy to present identity, and as signs that others on the site can read and interpret as they develop an understanding of the identity of others. Drawing on interview data conducted with users of the Livejournal SNS, this paper argues for a growing visual literacy among users of social networking sites.
\end{abstract}

Keywords: Goffman, Performance, Identity, Impression Management, Social Networking Sites.

\section{Introduction}

The rise of social networking sites has been well-documented in both the academic and popular literature. Much has been written about such sites [1], from the earliest BBS through to Facebook as the current SNS-de-jour, and how such sites are connecting individuals into communities of interest that may have little resemblance to make-up of their face-to-face, embodied social networks. However, much of the existing literature focuses on the textual nature of these SNS - 'words on a screen,' to paraphrase's Turkle's famous phrase [2]. Whilst text is still arguably the dominant element in facilitating online social interaction, platforms such as Facebook, Livejournal, and even Twitter include technological infrastructure that makes it easy (in many cases, simply point-and-click) and subsequent subtly encourage [3] users to include visual information, such as photos, either into their textual flow or onto the site that surrounds and frames the written exchanges.

This paper argues that such visual components contribute to the overall creation of a virtual identity, an online social self that engages with and is a part of the online virtual community, as part of the visual component's plural function in the online social space. By careful selection and control of these visual elements, individuals engage in a form of virtual impression management of their online persona or personas. Other participants in the online group can then read these visual cues and extract 
from them information that helps create an impression of the disembodied other with whom they are interacting. These visual cues can be read in isolation or as part of the overall semiotic structure of the online presence, to create their impression of the others' identity. This new semiotic richness is further enabled by the growing ease of use and sophistication of the tools that allow users to easily collect, manipulate, upload and engage with such images.

To support this argument for a growing visual sophistication in identity construction and performance among SNS users, this paper will draw on data collected during a study of Livejournal users regarding their use of images as part of their social activity on the site. This paper argues that in the relationship between users and SNS, users are becoming increasingly skilled not just in reading such visual information within a virtual social context, but also in the creation and deployment of such visual cues to help facilitate the development of particular virtual identities - that in fact these visual cues are being deployed as part of deliberate strategies of online impression management. This paper concludes by discussing some of the implications of visuality and impression management for the future of online identity formation and social group behaviour.

Firstly, however, it is necessary to explore further these notions of identity and impression management as they relate to online environments.

\section{Identity and Impression Management}

Identity as a concept encompasses a vast range of perspectives and ideas, and the disembodied nature of the internet further complicates issues of definition. In regards to online social networking, it may be useful to consider identity as the development of a biographical narrative that is constructed and reconstructed through relationships with the platform of interaction and with the wider social network within which it is situated [4, 5]. This definition, though not without its problems [6], encompasses the notion that identities, whether embodied or not, pseudonymous or not, have a history and a shared experience within their context [7] and deploy common signs and marker or props that helps enforce a consistent and decodable presentation of self within the wider social context. The construction and reconstruction of an identity narrative then becomes part of what Goffman [8] refers to as an everyday performance.

By taking this approach to notions of identity, the relation to issues of impression management becomes clear. Impression management is a broad term used in a number of fields to refer to the strategies and processes employed by individuals to influence how others perceive them [9, 10]. Impression management may be carried out for instrumental reasons, such as to evoke a particular response from others, or it may be used for more expressive means, such as creating a general presentation of self which would persist beyond a single encounter and which would both facilitate the social contact and create for others an understanding of identity that tallies in some way with the individuals own claimed or desired self-identity [11].

Though these two conceptions of impression management overlap significantly, it is this latter form of self-presentation that this article will primarily engage with. The works of Erving Goffman and his dramaturgical approach to identity and issues of impression management will form the basis of the analytical framework of this study. 
Such an approach to examining identity presentation in SNS is not novel [12, 13] [14], but this particular study will look at how users of SNS deploy visual cues as part of a performance of a persistent identity narrative that they construct as part of their online activity.

Impression management relies heavily on the specific context in which the social interaction is taking place. I would argue this to be especially true in online interactions. The 'rules of conduct,' to borrow the phrase from Goffman, are the obligations and constraints that frame the interaction. These may be explicit laws or moral obligations, or more implicit honorifics or acts of etiquette [11]. In an embodied interaction, these rules of conduct are part of the shared cultural and social context of the space. Online, however, the participants may be situated in quite different contexts - for a successful social interaction, and thus successful impression management, the rules of conduct must be clear to all parties in the virtual environment. Thus rules of 'netiquette' can be seen to be part of wider context in which impression management occurs. However, these 'rules' are not universal, but are different between groups and shift over time or with changing context [15]. Therefore, in considering online impression management strategies, it is important to remember the shaping effects of such 'rules of conduct.'

It is also arguable that online impression management strategies are influenced by the channel of communication used to support the social interaction. Different rules of conduct, and indeed different social connotations can be ascribed to similar behaviours on different channels of communication or different social networking sites (think for example the different activities one might expect to find on MySpace as opposed to LinkedIn). Again, to adopt Goffman's terminology, we choose different impression management strategies depending on the stage (or SNS channel) on which we are performing, the props we have available (or are allowed by the site architecture) to deploy, and the audience we are expecting to engage with. For an individual to develop an impression management strategy, they have to have some understanding of the other that they are trying to make an impression on (often by 'reading' the impressions or identity narratives already given out in a particular environment, and then modulating their own impression management strategies accordingly).

Goffman's work was originally based on face-to-face contacts, with all the subconscious cues (such as dress or body language) providing both supplementary identity information and feedback for the performance of a particular identity. But as Browning and Stephens note:

It was assumed that ICTs would provide less 'social presence' and would filter out essential cues for interpreting what was going on, and there would be less rich media than face-to-face communication. Yet there is a growing collection of studies showing that people can use 'lean' media for effective social interaction... [11, p. 69]

People using ICT, and particularly CMC, adapted their performance and their impression management strategies to the new medium. For example, in purely text-based environments such as BBS and IRC chat, participants used emoticons, a consensual set of symbols (such as :D and /ol) to reintroduce social and expressive information once conveyed by body language. As participants became conversant with the shared 
symbolic vernacular of these new virtual environments, they adapted their impression management strategies to take advantage of these new modes of expression [16].

It is the core argument of this paper that this modification of self-performance and impression management strategies to incorporate new visual modes of communication is driving the shifting visual literacy of online participants within the context of specific online social networking sites. With this claim in mind, this paper will now explore one example of the use of images in online identity and impression management.

\section{Impression Management and Visuals on Livejournal}

Livejournal [17] is a social networking site that has been running since 1999 that allows users varying levels of access to customize their page on the site depending on the type of account they hold (paid, ad-supported, or free). This customization includes the option to use pre-made or to develop their own unique backgrounds (known as layouts), and to have different numbers of small, 100x100 pixel images, referred to as userpics or sometimes as icons. Each user (who often uses the site under a pseudonym) can choose from the list of pictures they have uploaded a specific userpic for each separate post or comment they make on the site. This differs from other popular SNS such as Facebook, which only allow a user to associate one image with their account or username at any one time.

Beyond these varying visual customization options, Livejournal also supports the ability for a user to embed images, video or other visual content into their posts and comments, either through html coding, or through a WYSIWYG client built into the Livejournal user interface. Third-party clients also offer users different point and click tools to help them embed such content into their posts.

These three forms of visual content - layouts, userpics, and embedded content mean that Livejournal has the capacity to support a visually lush virtual environment. For this paper, the focus will be on how users understand these images (both the ones they create and the ones they see made by others) in terms of identity and impressions management strategies.

The follow data are from open-ended, semi-structured interviews with a subset of Livejournal users who participated in a wider study of the uses of images of Livejournal that was conducted in 2007. A link to a survey had been circulated among English-speaking Livejournal users using a variation of snowball sampling where those who had taken the survey then posted a link to all their friends on the site, and so on. These users then elected to click the link and complete the survey. Interview subjects were then selected at random from the survey respondents who had completed an optin section of the survey indicating their willingness to participate in follow-up interviews. All subjects were English-language speakers over 18 years of age who used Livejournal. Due to the sampling method (particularly self-selection during the snowball phase), and the relatively small number of interviews (survey: $N=610$, interviews: $\mathrm{N}=21$ ), it must noted that these results may not be indicative of the Livejournal community as a whole ${ }^{1}$, but instead may reflect the subset of the user group who

${ }^{1}$ Particularly the large Russian/Cyrillic language community who were excluded by the English-language nature of the study. 
actively use or consider their own image strategies and were motivated to complete the survey. That said, their responses still give a tantalizing insight into the image use strategies of those who can manipulate and work with Livejournal's capacity to support visuals.

The aim of the initial survey was to attempt to gauge the extent to which images are being used, in terms of both creation and consumption, but it is in the interviews where motivations and reading strategies are described by the users themselves. Because of this, and because only a relatively tiny number of survey respondents participated in the subsequent interviewers, this paper will concern itself solely with the interview data. The interviews were conducted via email, and the textual transcripts of the interviews were then coded around a small number of core themes.

All interviewees had been using Livejournal for between three and seven years, with the majority of them having been using Livejournal for approximately four years at the time of the interviews ${ }^{2}$. The interviewees reported a reasonably diverse range of self-described visual skills in both reading and creating images.

Whilst the interviews covered a wide range of issues regarding visuals and Livejournal, it is the discussions regarding identity and impressions management that have been excerpted for closer analysis. In particular, two key themes are relevant to the question of impression management, visuality, and online social networking how users manage their own visual performance, and how they read the visuals put forth as part of other's online performances. Interview data was coded under these two themes is excerpted below and discussed in terms of identity, performance, and impression management.

The first theme taken from the interviews relates to how the users select and control their visual performance in Livejournal to promote or maintain a certain desired facet of their online identity. It must be noted that throughout the interviews, it was clear that users found it hard to articulate and reflect upon their own identity construction practices as they pertain to images. This may be in part due to the 'backstage' nature of such identity construction practices, as opposed to the 'frontstage' nature of the interviews with an interviewer that was personally unknown to them.

Even so, the interviewees did betray some of their strategies for impression management, often as they compared the perceived strategies of others with their own. In this regard, the interviewees often began by noting the expressive impact of images in their Livejournaling activity. For example, one respondent noted:

“....I tend to associate different userpics with certain kinds of moods or to the same extent, to the kind of content of a specific post....and rightly or wrongly, assume that any readers of my LJ [Livejournal] will do the same!" Respondent \#11

Later in the interview, the same respondent elaborated further on this emotive connection in regards to their wider reading habits:

"...over time, I've come to associate specific userpics with certain members of my F-list [friendslist].... Quite often, I scan my list to see who has updated, and will whiz by icons that I don't recognise (those

\footnotetext{
${ }^{2}$ Interview excerpts have been included 'as is' including typographical and grammatical errors. Editorial clarification is provided as needed in [square brackets].
} 
used by LJ users posting in a community, for example), and will stop at ones that are familiar to me - those of my friends. Occasionally this backfires as another user may have the same icon as one of my LJ friends and for a second I'm confused as to why I don't immediately recognise the writing style or contents of the post." Respondent \#11

Similar comments were made by a majority of the interview subjects, and it was one of the dominant themes of the interview overall. In terms of images as semiotically rich means of communicating identity and helping to manage their impression, two ideas are suggested. Firstly, that images, particularly images appended to accounts such as userpics/icons, can be read in multiple ways simultaneously - as both identity marker for the account overall (associating particular userpics with particular otherwise disembodied users), and as additional information relevant to the specific post (association with moods or content). This may be specific to Livejournal, where the architecture of the site enables the user to select userpics off a pre-loaded list for each post or comment, but also has implications for readership on sites that force a more static visual code onto their users.

Userpics, and to a lesser extent, layouts, are also seen to be part of a larger strategy of online identity and impressions management that transcends the boundaries of a specific SNS, and even the distinction between the virtual identity and community and the embodied identity and community of the user. For example, these three quotes are from three different interviewees, but all play on the common theme of consistency in the visual construction of an identity narrative in a social space.

"I mainly use a very recent photo of myself as my default icon! I feel, personally, that it's important that I BE who I am - to the extent that I can be - online. Of course, I want it to be the most flattering photographic representation, yet I feel it needs to be authentic." Respondent \#15

"I stick to one icon, which I tend to use on lots of different services (i.e.; My LJ icon is also my Facebook image, or at least they're both from the same photo)....I want my different accounts in different places to be seen as parts of a whole. I only use different services because of what they provide, not to present different versions of myself." Respondent \#20

"I had my own icon with my LJ name on it and yellow daffodils. When I deleted it [old LJ account], I really missed the icon too. A friend of mine made a new one with my new LJ name and yellow daffodils. The funny thing is that when I commented on other people's journals some of my old 'friends' recognized me, even if my LJ name is very different now. I guess I am the one with the daffodil icon. "Respondent \#6

For these respondents, the visual information conveyed in their userpics is part of a wider strategy of identity association that persists across time and even SNS platforms, and which continues despite changes in username or account name, which would be assumed to be a primary source of persistent identity in cyberspace. The user image can, in very limited ways, be thought of as analogous to a face, a 
consistent and instant visual identity cue (however, care must be taken not to overextend this metaphor). Users select and maintain certain image associations over time, even cultivating otherwise generic visual cues (such as with the daffodils) to indicate personal identity within specific social groupings. These images become 'owned' in the sense that they are invested with the shared history developed along with the image's usage - they in effect become a marker of a persistent, recognized identity.

Of course, for persistent identity management strategies to function, they need to be read by others. This leads into the second theme that arose in the interviews, how others engaged with these identity cues and management strategies.

The interviewees were able to far more easily articulate their strategies for reading and understanding the use of visuals and images by other users (perhaps, again, because reading is also a frontstage act). They highlighted two key elements that recurred in how they see others using visual in Livejournal - for identification, and to decode personality traits.

As noted earlier, it was at first thought that social interactions conducted online using services such as SNS would have a thinness, a reduced 'social presence' as the mediated and virtual nature of the connection stripped out the non-verbal cues such as expression and body language, dress and deportment. Yet these interviews, and similar studies on other online cohorts [18] suggest that these users are beginning to put these cues back into their online interactions by using specific visuals elements as markers of a particular identity performance. This tallies with the dual encoding of information interviewees seemed to suggest as noted above - that alongside contextspecific markers of mood or relationship, images also functioned in a more general sense as a quick way of saying within the shared social space that 'this is me.'

For example, the interviewees often made comments such as:

"I have difficulty matching images to names, so if people are frequently changing their icons, I can't use them for identification. I have to go find the username written somewhere on the screen instead. "Respondent \#20

"I also most identify people on LJ with one or more userpics - I think people in general so visually orientated that it's tough to NOT associate the user with some kind of visual at some point." [original emphasis] Respondent \#14

This last comment is particularly interesting, in that it suggests a substitution behaviour between online and offline identity strategies - this notion of substituting the face/body and associated non-verbal cues with a small set of static images was implied by a number of the interviewees. One interviewee went further:

"[visuals like userpics are]...little non-verbal clues that are so sorely missing in pure text; to me, they are online equivalents of body language and other secondary visual cues." Respondent \#13

This user made a direct and explicit correlation between visuals and substitute nonverbal cues - however, they also ranked such cues secondary to the textual communication, the words of a screen, which were also emphasized by many respondents to be the key information on the page. 
The identifying use of images went beyond mere association over time. An even more prevalent theme was the assumptions these users made about other users based on the visual content of their journals. It is here that issues of impression management, particularly in terms of those knowing who are the intended recipients of the impression management strategy, come to the fore.

Users infer a lot about other users through their use (and perceived 'misuse') of visuals, especially layout designs and embedded visuals. Many of their readings of such visuals seemed based on ingrouping and outgrouping judgments - members of 'my' group have this aesthetic and use these visual elements in these ways, and members of that group use other elements. For example:

"A highly personalised layout conveys the information that the user is highly computer literate (unless there is a credit to someone else) Sophisticated or low key, understated layouts convey that the user is older and perhaps more educated (if reflected by content) Bright pink layouts with Hello Kitty pictures convey that the user is likely to be younger/not sophisticated. Often choice of layout gives an idea of whether the user may be quite extroverted or introverted. Nondescript layouts are less informative but could convey that the user is either not very engaged with lj at all, or not very visually orientated.” Respondent \#7

"If their icons all blink, move, or are animated, i am inclined to think they are selfish and pushy--they are forcing their annoying "lookat-me, look-at-me" icon into others' space. If their icons are all anime, or television-derived, i'm inclined to think they are disinterested in real sharing, fundamentally uninteresting, and probably shallow. if they post a lot of memes that have graphics, i'll come to the same conclusion--they're uninteresting. On the other hand, if they post updates of their lives with photos for illustration, i feel i'll understand them better because i can *see* a little glimpse of what's going on--what their space is like, what they look like (a face behind the words), what they're up to." Respondent \#12

"I tend to immediately like people who reference cultural artifacts (books, movies, etc) I like in their icons." Respondent \#16

In this sense, these visual might be better considered not in terms of an isolated individual identity, but as part of a social identity that the individual user cultivates and manages using such images and design strategies as appropriate to that social group to declare 'this is where I belong.' One interviewee was able to articulate more fully these multiple purposes of images in regards to an individual's plural identities:

"Some icons are used to indentify [sic: identify] with a particular community, others are more generic. Some posters use one selfidentifying icon all of the time, allowing them to move between communities with ease. Photos and artwork often open dialog in communities, which can be fun and interesting, or divisive and 
controversial. It depends on the content. Overall, I believe that visuals in any form are part of who we are on LJ as people and as individuals in larger groups." Respondent \#17

Userpics in particular often appear to function as a marker of affiliation by drawing on source materials and visual tropes and cues specific to particular subgroups. Tarkowski, in his analysis of userpics and peer production, also highlights the intersection of cultural and individual identity and notes the influence of popular culture in these images [19]. As noted earlier, these users seem to make these image do the work for multiple roles: identity marker, a claim to social belonging, a reference to a shared interest or popular culture icon, a sign of a mood or even a denotative part of the text (i.e.: "this is the kind of dress I'm talking about).

The strategic deployment of images within a specific social group and through a channel with particular technological capabilities involves a multi-leveled understanding of the role such images play in context. A single image can be simultaneously a marker of identity, a signal of in-grouping, and an expression of emotion or context. Rebaza noted a similar multiplicity of meanings in Livejournal userpics [20]. Other users then decode (or fail to decode) one or more of these layers in ways that make sense to them, and from repeated interactions and continuous cycles of coding and decoding of visuals alongside text and other content, slowly develop an understanding of each other in the virtual environment.

\section{Discussion}

Though these interviews dealt mainly with the interviewees own perceptions of their visual reading and writing strategies in their own online social contexts, their comments suggest some interesting implications for the future of user-generated multimedia and social networking and strategies of online identity and impression management.

Firstly, as in other forms of impression management, understanding the rules of conduct as pertains to a particular social network or community is vital to creating an impression strategy that will be read positively or as intended. As many interviewees emphasized, certain aesthetic preferences, colour palettes, or subject choices led them to read the sender of such images in certain ways, some more desirable than others young, immature, of like mind, etc. A recurring theme across the interviews was how quickly a new contact could be disregarded if their presentation included undesirable images or aesthetic in layouts or userpics. By knowing the rules, and more importantly, in having the visual skills to engage with and conform to those rules in ways that were still unique, a user could present an identity that would garner a positive reaction and thus further engagement from others in the network. In other words, they had successfully used images to communicate their understanding of the 'rules of conduct' for that social group.

Secondly, it must be reiterated that the technological platform and channel of communication (in this case, Livejournal) also has significant impact on the strategies chosen by virtue of the kinds of visual control it allows - from differing numbers of userpics depending on account type, through to varying levels of sophistication in layout design depending on whether a user can (either themselves or through the 
agency of others [21]) code a new CSS layer, or whether they have to use an existing skin or default layout. Image creation and manipulation (using tools such as Photoshop $^{\mathrm{TM}}$ or Gimp) also limit the level of visual information a user might code into their displays. Different levels of social currency are ascribed to different skill sets by different groups, based upon the priorities of those groups. Alongside this, crossplatform and cross-channel consistency in visuality is also increasing in importance as some users seek to build a consistent visual identity strategy across multiple SNS, such as Facebook and Livejournal. That said, different platforms with different visual capabilities may enable different strategies of visual self-expression. For example, Facebook, which is now arguably the biggest single repository of photos on the web [22], only allows a single user image which is appended to all text, yet visual expression is still enabled through referring to user photo albums and even the visuals associated with quizzes and other elements which appear on the livefeed of all users. This architectural framework then forces different visual strategies than those that might have been used on other SNS such as Livejournal.

Thirdly, and perhaps most importantly, for visual to have such value in online impression management as these informants seem to imply it does, then there must be some level of online shared symbology for these more complex images, just as there is for the emoticons that evolved in text based exchanges. The multi-layered nature and richness of the visual elements in userpics, layouts and in-text visuals does leave them open to more diverse interpretations but I would argue that, within a specific context (an in-group using a particular platform) there is a consistency of image reading that is part of in-group membership. Thus visuals that employ these in-group visual cues not only convey emotional and identity information about the discrete individual, they also contribute social information about the place of that individual in the network. This, I argue, is partly why users can immediately discard certain user sites as 'immature,' 'unsophisticated' or 'shallow' - they are not conforming to the implicit in-group rules of reading and writing imagery, and thus indicate a lack of shared history or trust. Through this, skills of visual creation, manipulation and deployment become an increasingly important part of an overall individual and social impression management strategy in web 2.0 environments.

These strategies of reading visuals, and of writing visuals to be read in desired ways, is becoming an important part of overall strategies of impression management for users of social networking sites that allow such visual expressions of self. By manipulating visuals alongside text, users can help 'flesh out' their online selfpresentation with personal, social, emotive and indicative information that other users can read (with varying levels of sophistication) to develop their own understanding or impression of that identity, and how it relates to their own in the online social context.

To conclude this paper, I would like to outline some implications of the above research and suggest possible future research directions in this area.

Firstly, though limited by its sample and scope, this research suggests that, at least among these users using this platform, there is a growing level of sophistication in the reading and deconstruction of images as they pertain to online identity. I would suggest that this may be due in part to both the increased capacity for easier inclusion of visuals on this platform, and to a growing expectation from users to have a visual component to the online self-presentation (whether it be a unique userpic, a personalized layout, or something more creative). This then raises questions as to whether this 
behaviour is unique to sites like Livejournal (which foster such a diverse range of visual customization) or whether equivalent behaviours can be found on more visually consistent sites such as Facebook.

Secondly, and perhaps more contentiously, I would argue that the desire to create a positive impression in online social networks using visuals is in part helping to drive an overall increase in visual literacy amongst those users. This research indicated that these users had developed their own skills (in what might be considered an 'amateur' rather than 'amateurish' [23] way) in terms of being able to manipulate and use images as part of their online social interactions. I would argue that this growing sophistication is being driven by a desire to present the best virtual 'face' in online engagements. Because there is such a personal need to use visuals effectively, I argue that users feel motivated to develop visual skills, whether that be in terms of aesthetics, manipulation, or deployment (such as with CSS code). Furthermore, the nature of Web2 social networks creates an environment where users feel empowered to learn from each other techniques for visuals, thus driving an ever-widening recursive loop between images presented and the meanings associated by the social group to such images. As one respondent notes regarding how they believe their skill in creating visuals has changed over time:

"I can 'see' how other artists do something new in a piece and it spurs me on to try the technique in my own way. Sometimes their advances create a jumping off point for my own and I'm able to develop something new from their inspiration.” Respondent \#17

Such sharing of techniques and visual approaches can also be seen on Livejournal where those interested in creating layouts, userspics or other site-specific visuals often forms communities such as userpic-sharing communities, or LIMS ('Last Icon Maker Standing") communities where creators share ideas and even have 'competitions' to create images along certain themes. Such competitions, and the more informal evaluation of images that appears to be part of the overall experience of social activity on the site, suggest a kind of 'appreciation' is going on where, if images are used, they have to be 'good' images within the overall calculus of identity evaluation that occurs in this virtual space. This then returns to an earlier point about the multiple layers of information - social, informational, personal - that can be contained within the image that is deployed within a social context. However, this research only scratched this surface of this layering function of identity images, and it is an area that would benefit from further research.

\section{Future Implications}

Though this research looked at image use in one particular web 2.0 platform, it does open up questions for the use of images across the social web. Firstly, with different platforms enabling different forms of visual expression (multiple versus single userpics, embedding images, customizing layouts), will skills in creating and understanding such visuals change as different platforms obtain dominance in the social networking cyberscape? If visuals are to become a part of the everyday function of social networking sites, will people develop sophistication in creating images as well 
as reading them, or is the experience of these Livejournalists and their 'amateur' visual creation an aberration?

Beyond this, and given the growing plurality of sites and services offering social networking for various groups, is it feasible to expect a 'universal' visual lexicon (such as evolved with the core group of emoticons), or are visuals too layered and too open to multiple interpretations to expect widespread commonality in creating and interpreting identity-related visuals? Or is it, as these respondents seemed to indicate, related more to the context in which the image is created, produced, disseminated and finally read.

\section{References}

1. boyd, d.m., Ellison, N.B.: Social Network Sites: Definition, history and scholarship. J. Computer Mediated Communication 13(1) (2007), http://jcmc. indiana. edu/vol13/issue1/boyd.ellison.html

2. Turkle, S.: Life on the Screen: Identity in the Age of the Internet. Simon \& Schuster, New York (1995)

3. Skog, D.: Social Interaction in Virtual Communities: The significance of technology. International Journal of Web Based Communities 1(4), 464-474 (2005)

4. Liu, H.: Social Network Profiles as Taste Performance. J. of Computer-Mediated Communication 13(1) (2007), http: / / jcmc. indiana. edu/vol13/issuel/liu.html

5. Hardey, M.: Life Beyond the Screen: Embodiment and identity through the internet. The Sociological Review 50(4), 570-585 (2002)

6. Ladd, K.: Textuality, Performativity and Archive: Examining the virtual body in socially networked space. MA Thesis, University of Toronto (2009), http: / hdl . handle.net/1807/18093

7. Donath, J., boyd, d.m.: Public Displays of Connection. BT Technology Journal 22(4), 7182 (2004)

8. Goffman, E.: The Presentation of Self in Everyday Life. Anchor, New York (1959)

9. Leary, M.R., Nezlek, J.B., Downs, D., Radford-Davenport, J., Martin, J., McMullen, A.: Self-Presentation in Everyday Interactions: Effects of target familiarity and gender composition. J. of Personality and Social Psychology 67(4), 664-673 (1994)

10. Panteli, N., Duncan, E.: Trust and Temporary Virtual Teams: Alternative Explanations and Dramaturgical Relationships. Information Technology and People 17(4), 423-441 (2004)

11. Browning, L., Stephens, K.K.: Impression Management and ICTs. In: Browning, L.D., Steinar Saetre, A., Stephens, K.K. (eds.) Information and Communication Technologies in Action, pp. 65-72. Routledge, New York (2008)

12. Marwick, A.: I'm a Lot More Interesting than a Friendster Profile: Identity presentation, authenticity and power in social networking services. Paper Presented at AoIR IR6.0: Internet Generations, Chicago, IL (2005), http://www.tiara.org/papers/ marwick_friendster_authenticity_power.doc

13. Kendall, L.: Shout Into the Wind, and It Shouts Back: Identity and interactional tensions on Livejournal. First Monday 9(3) (2007), http://www.uic.edu/htbin/ cgiwrap/bin/ojs/index.php/fm/article/view/2004/1879

14. Pearson, E.: All The World Wide Web's A Stage: The performance of identity in online social networks. First Monday 3(2) (2009), http://firstmonday.org/htbin/ cgiwrap/bin/ojs/index.php/fm/article/view/2162/2127 
15. Fono, D., Raynes-Goldie, K.: Hyperfriends and Beyond: Friendship and social norms on Livejournal. In: Consalvo, M., Haythornwaite, C. (eds.) Internet Research Annual, vol. 4, pp. 91-118. Peter Lang, New York (2007)

16. Marwick, A.: Selling your self: Online identity in the age of commodified Internet. University of Washington, Washington (2005) (Unpublished MA Thesis),

http: / / www.tiara.org/papers /

amarwick_sellingyourself_mathesis.doc

17. Livejournal, http://www. livejournal.com

18. Siibak, A.: Constructing the Self through the Photo selection - Visual Impression Management on Social Networking Websites. Cyberpsychology: Journal of Psychosocial Research on Cyberspace 3(1) (2009),

http: / / cyberpsychology.eu/

view .php?cisloclanku=2009061501\&article $=1$

19. Tarkowski, A.: Peer production of popular culture at the Live Journal blogging site. Banal blogging or cultural struggle? Presented at RE: Activism: Re-Drawing the Boundaries of Activism in New Media Environments. Budapest (2005),

http://mokk.bme.hu/centre/conferences/reactivism/ submissions/tarkowski

20. Rebaza, C.: Online Gestures: Icon Use by Fan Communities on Live Journal. Paper Presented at 41st Hawaii International Conference on System Sciences. Hilton Waikoloa Village Resort, Waikoloa (2008)

21. Pearson, E.: Digital gifts: participation and exchange in Live Journal communities. First Monday 12(5) (2007),

http://firstmonday.org/issues/issue12_5/pearson/index.html

22. Wong, P.: Conversations About the Internet \#5: Anonymous Facebook Employee. The Rumpus (2010), http://therumpus.net/2010/01/conversations-aboutthe-internet-5-anonymous-facebook-employee / ? full=yes

23. Lessig, L.: TEDTalks: Laws That Choke Creativity [online video file] (2007), http: / /www.ted.com/talks/larry_lessig_says_the_law_ is_strangling_creativity.html 